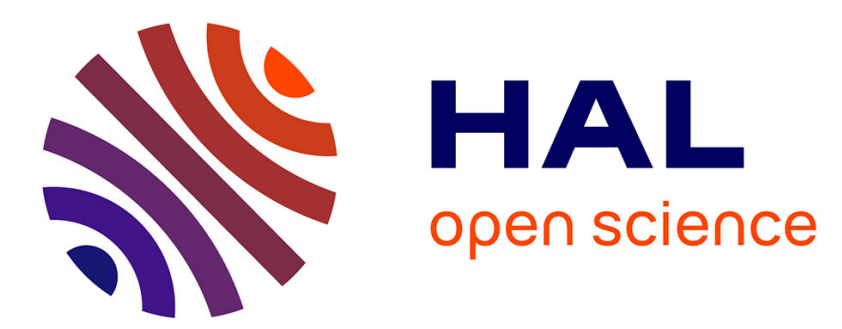

\title{
Les carrières de La Lie à La Roche-Vineuse (Saône-et-Loire) \\ François Cognot
}

\section{To cite this version:}

François Cognot. Les carrières de La Lie à La Roche-Vineuse (Saône-et-Loire). Gallia - Archéologie de la France antique, 2002, Carrières antiques de la Gaule, 59, pp.121-131. 10.3406/galia.2002.3100 . hal-01912494

\section{HAL Id: hal-01912494 \\ https://hal.science/hal-01912494}

Submitted on 20 Jan 2020

HAL is a multi-disciplinary open access archive for the deposit and dissemination of scientific research documents, whether they are published or not. The documents may come from teaching and research institutions in France or abroad, or from public or private research centers.
L'archive ouverte pluridisciplinaire HAL, est destinée au dépôt et à la diffusion de documents scientifiques de niveau recherche, publiés ou non, émanant des établissements d'enseignement et de recherche français ou étrangers, des laboratoires publics ou privés.

\section{(이) $\$$}

Distributed under a Creative Commons Attribution - NonCommercial - NoDerivatives $\mid 4.0$ 


\author{
François COGNOT
}

Mots-clés. Carrière, calcaire pisolithique, habital, sarcophage, personnage sculpté.

Key-words. Quarry, pisolitic limestone, settlement structures, sarcophagus, human sculpture.

Résumé. À moins de $10 \mathrm{~km}$ à l'ouest de Mâcon, le banc de calcaire pisolithique de La Lie (commune de La Roche-Vineuse, Saône-etLoire) a été exploité en carrière. I) $I^{r r}$ s. au XX s., périodes d'activité et d'abandon se sont succédé. Ies extensions ont dégagé, par étapes, sur le flanc de la colline, une surface de plus de 2,5 ha et un front de carrière de plus de $20 \mathrm{~m}$ de haut dans lequel des galeries ont été creusées. La pierre, tendre à demi-ferme, peu gélive, se prête volontiers à une taille fine et adoucie. Les études entreprises depuis 1994 ont conduit à meltre au point une méthodologie spécifique applicable aux carrières, permettant de déterminer les techniques d'extraction, as types de blocs extraits et leur probable destination, la progression des chantiers successifs, la différenciation des périodes d'extraction. Y,es recherches ont révélé la présence d'habilats, à l'intérieur du périmètre des carrières. Des gravures pariétales sur front de taille $\| l$ to un personnage sculpté sur une laisse de carrière ont été mis au jour.

A sstract. A bedrock of pisolite limestone, just a few kilometres west of Mâcon, has been exploited at La Lie (commune of La Roche Vineuse). 1) iring twenty centuries of exploitation, quarrying was fluctuant and ceased from time to time. The operations extended on the hillside on a surface area which exceeded 2,5 ha. Calleries were carved out of the quarry face which raise to a height of over $20 \mathrm{~m}$. The soft Mimestone bedrock does not show frost rracking and is pasy to zuork. The application of a new method "Stone Quarry Archaeology" during excavations since 1994 has brought into light different aspects of the quarrying techniques - cutting and typology of blocks -, as well as the development of workshops, and the successive stages of activity have been identified. Stone structures are attested within the quarrying area and other finds were uncovered, rock-engravings and the relief-carving of a human figure on a free standing stone.

\section{SITUATION}

Les carrières de La Lie ont été ouvertes à la partie sommitale du flanc ouest d'une colline (point culminant $313 \mathrm{~m}$ ). Propriété de la commune de La Roche-Vineuse, elles dominent les hameaux, en partie imbriqués, de Somméré et des Bouteaux. L'ensemble se trouve à $10 \mathrm{~km}$ à l'ouest de Mâcon et à $15 \mathrm{~km}$ de Cluny (fig. 93).

La zone exploitée s'étend sur près de $300 \mathrm{~m}$ du nord au sud et de 55 à $150 \mathrm{~m}$ d'est en ouest. Les extractions successives visibles s'étagent sur une hauteur de plus de $20 \mathrm{~m}$ au nord-est. Mais les déchets de taille laissés sur place, les remblais et comblements réalisés au fil des siècles ont recouvert le sol de carrière principal. Ainsi, tout le front de taille ${ }^{*}$ ouest est dissimulé. Le sol de carrière est actuellement totalement invisible, enseveli sous une épaisseur de déchets estimée à $8 \mathrm{~m}$.

\section{INTÉRÊT ARCHÉOLOGIQUE}

L'intérêt du site, déjà mis en évidence dans les années 1980 par A. Barthélemy (Barthélemy, 1984), dépasse le cadre local :

- la réunion en un même lieu et sur une surface très étendue de différents modes d'exploitation (à ciel ouvert et en galeries) d'une part, de périodes d'exploitation 


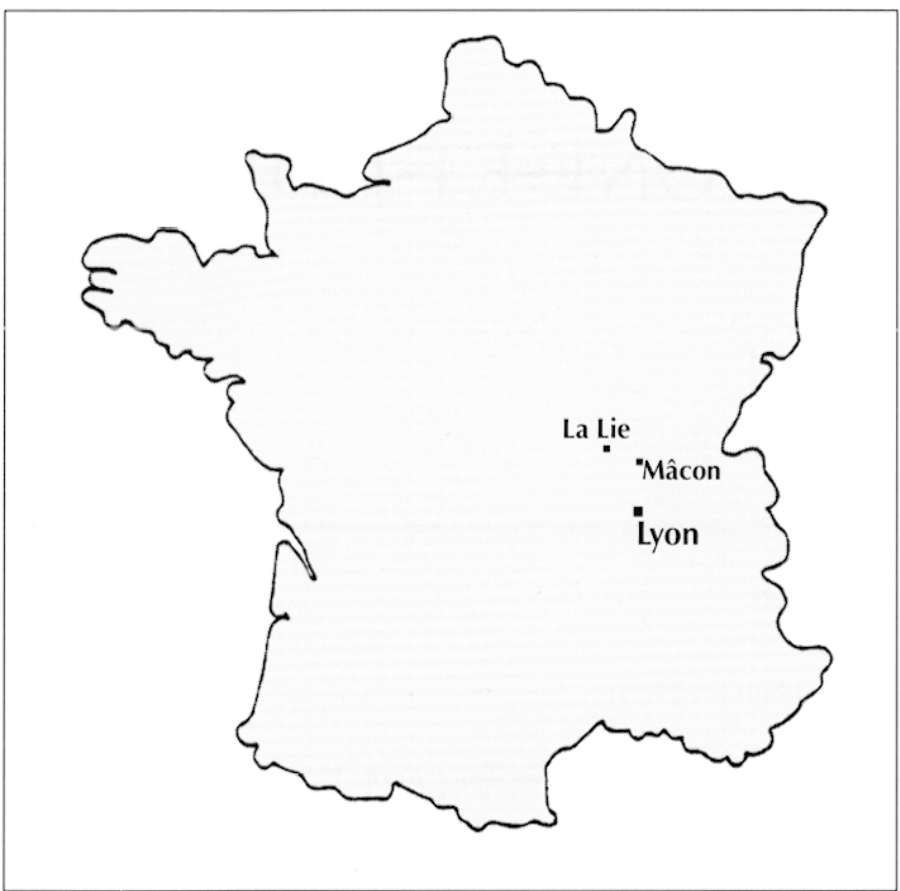

1

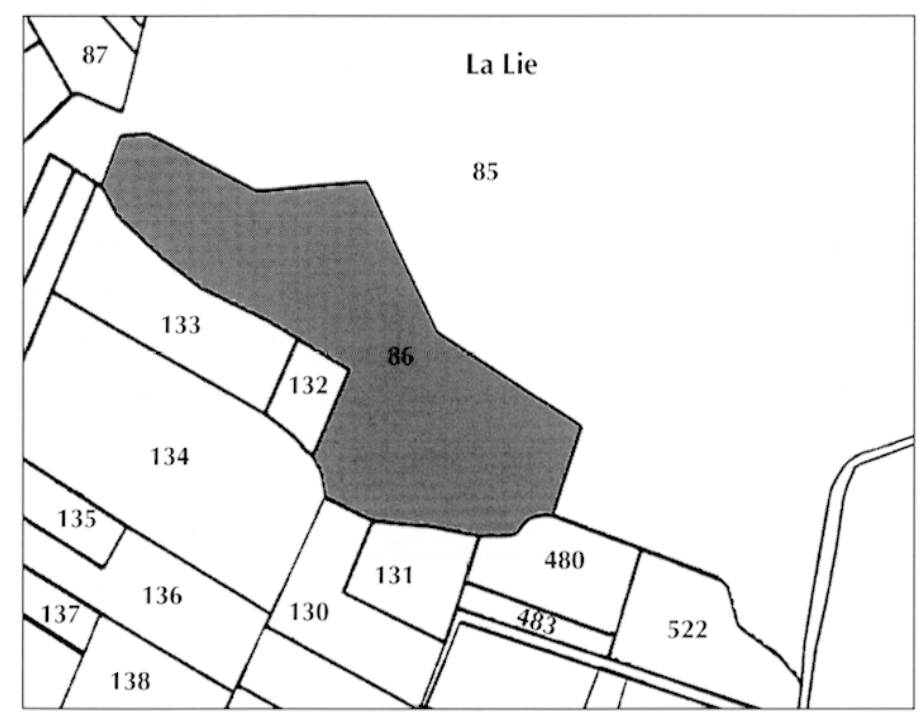

3

nettement caractérisées, étalées entre le $\mathrm{I}^{\mathrm{er}} \mathrm{s}$. et le $\mathrm{XX}^{\mathrm{c}} \mathrm{s}$. d'autre part, est exceptionnelle ;

- pour chaque période historique constatée, le lieu offre une potentialité d'informations nouvelles et originales ;

- des éléments touchant l'histoire de l'art (dessin, esquisse, gravure, sculpture, ébauche, etc.) et l'épigraphie (dédicace, compte d'exploitation, etc.) viendront s'ajouter aux connaissances déjà acquises.

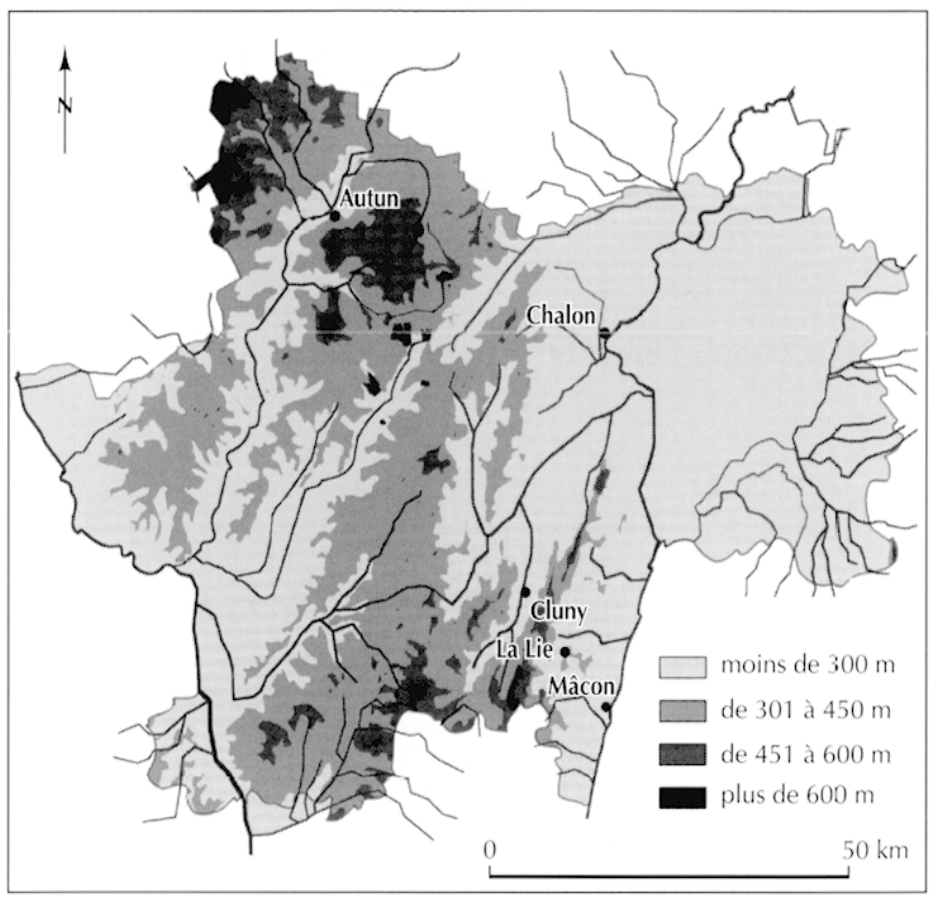

2

Fig. 93 - Localisation géographique des carrières de La Lie : 1, carte de France; 2, département de Saône-et-Loire (d'après F: Teinturier et B. Chevalier) ; 3, plan cadastral de La Roche-Vineuse, section li, 1982 (I)AO F. Cognot, Maison de l'archéologie, Mâcon).

\section{LIMITES DE L'ÉTUDE : \\ LA CARRIËRE NORD}

Les travaux entrepris depuis 1994 ont permis de distinguer trois grandes zones de carrière mais seule la première, dite carrière Nord, est circonscrite. L'essentiel des études réalisées porte sur celle-ci (fig. 94). Elle a été exploitée à ciel ouvert" et en galeries. 

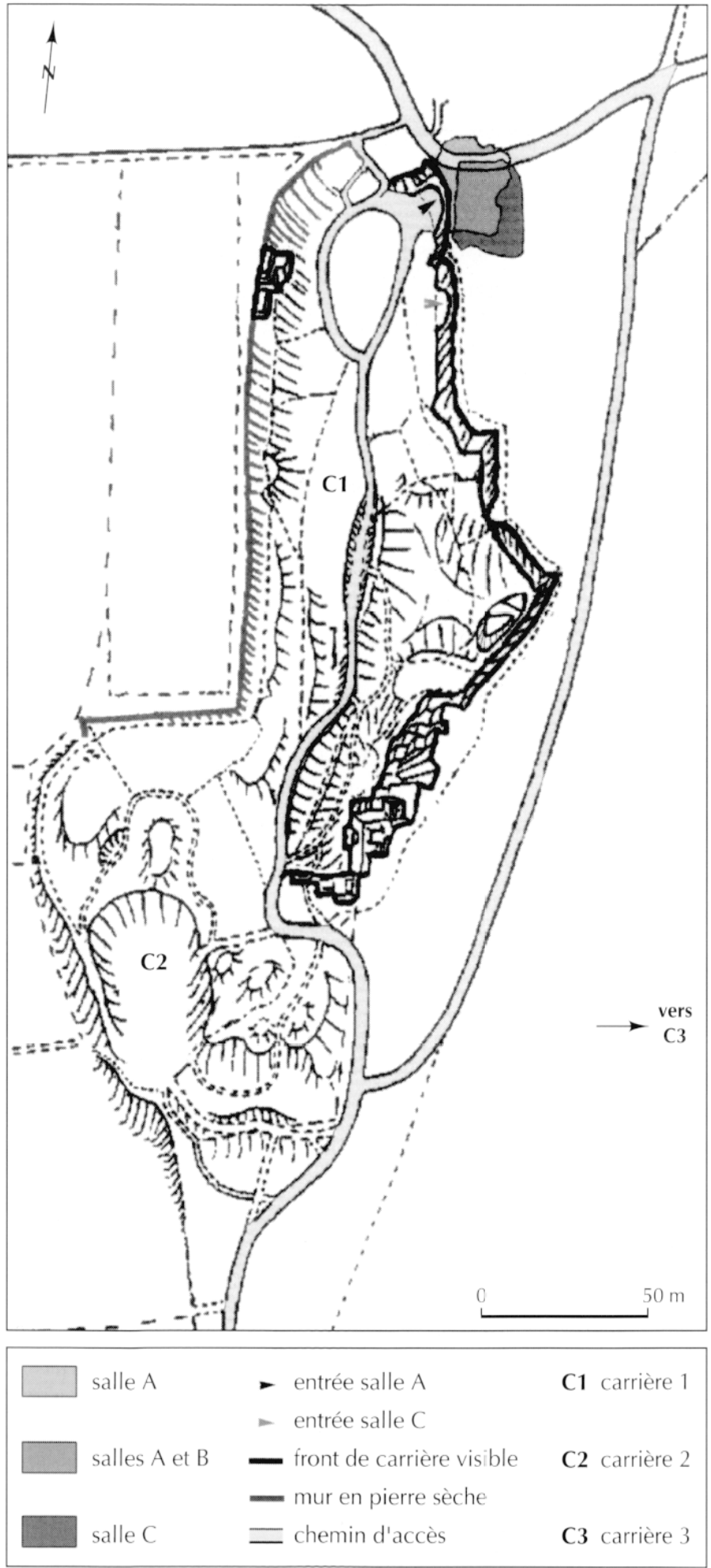

Fig. 94 - Plan général de la carrière 1 aver superposition des salles A et B (dessin F: Cognot, Maison de l'archéologie, Mâcon).

La partie aérienne s'inscrit dans un rectangle de $200 \mathrm{~m}$ (du nord au sud) par $55 \mathrm{~m}$ (d'ouest en est). Un triangle approximativement isocèle de $20 \mathrm{~m}$ de hauteur

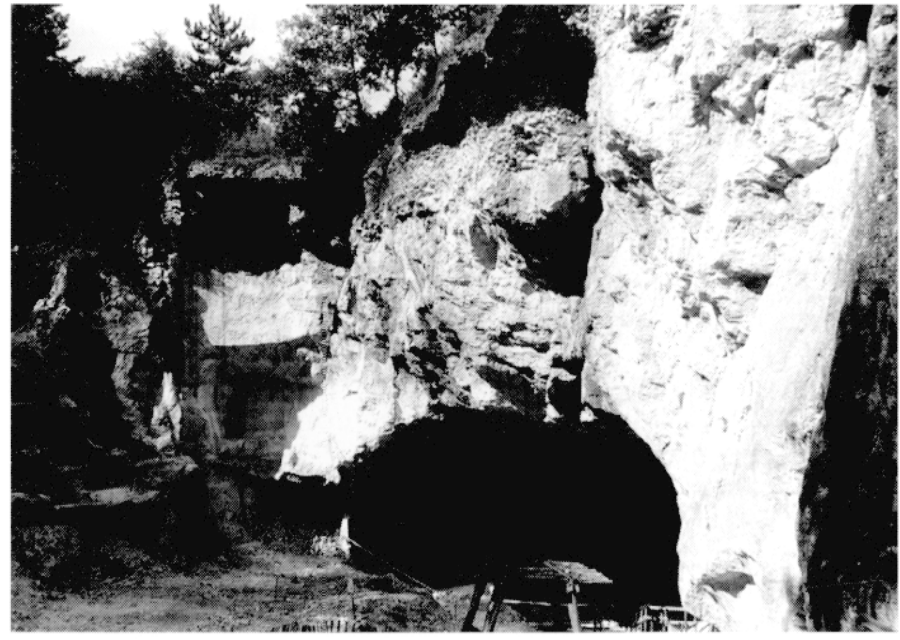

Fig. 95 - Vue du front de carrière nord-est avec la salle A dite grande cavité (photo I. Cognol, Maison de l'archéologie, Mâcon).

vient s'appuyer par sa base (environ $100 \mathrm{~m}$ ) sur la partie médiane de la longueur est. Le front de carrière* occidental est totalement recouvert tandis que le front oriental est en grande partie visible.

Des galeries d'exploitation ont été ouvertes dans les fronts de taille*, depuis la partie à ciel ouvert, formant des cavités étagées en hauteur (fig. 95 et 96). Actuellement cinq d'entre elles ont été reconnues au nord-est et au nord (salles A à E), mais d'autres, pénétrant sous le front de taille à l'est, sont encore inaccessibles. La salle A (dite grande cavité) est partiellement superposée à la salle B (dite salle souterraine : inventée le 27.01.1995). Au sommet du triangle, côté oriental, un ensemble de rochers, d'un volume d'environ $3000 \mathrm{~m}^{3}$, marque sans aucun doute une galerie d'exploitation dont le ciel" s'est effondré.

\section{GÉOLOGIE : LA PIERRE DE LA LIE}

Si la proximité de voies de communication a pu être un critère dans le choix de l'implantation de l'exploitation, il n'en reste pas moins que c'est le matériau même qui était principalement attractif et recherché. Examiner les caractéristiques géologiques du gisement conduit à mieux saisir l'intérêt qu'il a suscité à toutes époques, et mieux circonscrire les fins et formes diverses de son emploi.

L'ensemble appartient à l'ère mésozoïque secondaire, période du Jurassique supérieur (Malm), étage 
oxfordien (Perthuisot, 1967, p. 36). Adrien Arcelin le situe dans un étage corallien (Arcelin, 1881, p. 206-210).

L'Oxfordien terminal (dit calcaire de Domange, unité $\mathrm{C}$ des géologues) est présent à $\mathrm{La}$ Lie en strates calcaires lithographiques dont l'ensemble a une épaisseur moyenne de $9 \mathrm{~m}$. L'affleurement du banc massif laisse apparaître une pierre beige à patine blanche. Très dure et résistante, elle est naturellement fracturée. Flle peut être employée comme moellon pour des constructions en petit appareil assisé.

Sous ce banc, les deux autres couches de calcaire lithographique et sublithographique - l'une de couleur rose saumon à brun, l'autre de couleur beige, de structure grumeleuse - sont débitées en plaquettes fines. Très gélive, cette pierre se détache des fronts de taille lors de chocs thermiques, essentiellement au printemps. Elle est impropre à la construction, sauf si elle est employée en remplissage entre deux parements et noyée dans un liant (mortier à la chaux ou béton).

L'Oxfordien supérieur (dit calcaire de Somméré) est décomposé en deux unités $\mathrm{B}$ et $\mathrm{A}$ par les géologues. L'unité B (partie supérieure), d'une épaisseur moyenne de $4 \mathrm{~m}$, se compose de deux strates de calcaire pisolithique et d'une strate dolomitique intercalée formant un banc repère en relief. La pâte fine des couches pisolithiques a une plus ou moins forte teneur en oxyde de fer. Le calcaire dolomitique est plus dur et cassant. Sa cassure est conchoïdale et accentuée ponctuellement par des conchoïdes dispersées dans la masse. Elle peut convenir à la construction car elle est très résistante, mais ne peut être travaillée finement.

L'unité A (partie inférieure), d'une épaisseur visible de $16 \mathrm{~m}$, est essentiellement le niveau exploité par les carriers. Elle est formée d'une seule couche de calcaire pisolithique tendre à demi-fermc, compact et onctueux au toucher, presque crayeux, de teinte allant du blanc franc au rose saumon, avec des pisolithes de grande taille. Facile à tailler, la pierre peut être affinée et adoucie mais elle est résistante au gel. Après vieillissement, un calcin protecteur lui donne une teinte allant du blanc crème au brun-rose. Le banc est fissuré de diaclases parallèles et perpendiculaires, subverticales. Larges de 10 à $50 \mathrm{~cm}$ au niveau supérieur de la couche de calcaire pisolithique exploitée, et plus ou moins comblées d'argile de décomposition, elles se rétrécissent jusqu'à ne plus être qu'une simple ligne brun-rouge, marquée par une plus forte concentration d'oxyde de fer. Elles disparaissent en majorité au niveau moyen du banc. Cette caractéristique de la roche a été mise à profit par les carriers de la même façon qu'ils ont utilisé à l'occasion les joints de stratification.

Les couches géologiques ont une pente orientée approximativement nord-est/sud-ouest. L'érosion des terrains a enlevé toutes les couches supérieures (unité C, et une grande partie de l'unité B) au sud de la carrière 1, rendant très accessibles les couches pisolithiques exploitables. En revanche, au nord, des terrains géologiques (plus récents que l'unité $\mathrm{C}$ ) sont demeurés en place. Cela explique en partie pourquoi, au nord et au nord-est de cette carrière, l'exploitation s'est largement développée en galerie.

\section{LES PRODUCTIONS DE LA LIE}

L'analyse des empreintes de blocs, sur les fronts de taille $^{*}$ et les sols de carrière actuellement dégagés, n'en est qu'à ses prémices. Elle est obligatoirement partielle car seuls les éléments visibles sans fouille peuvent être étudiés. Les autorisations d'intervention délivrées n'ont été jusqu'à aujourd'hui que des autorisations d'inventaire ou de sauvetage. Cependant, il est déjà possible d'avoir un aperçu général, mais provisoire et partiel, de la production selon les différentes époques d'exploitation et de distinguer les phases majeures de production. Les traces d'outil, la forme des tranchées d'extraction, la présence ou non d'emboî-

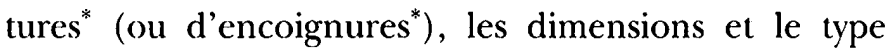
d'enlèvement sont autant d'éléments qui vont permettre d'établir une chronologie relative de la progression et la succession des chantiers.

L'exploitation des carrières de La Lie a débuté au $\mathrm{I}^{\mathrm{er}}$ s., elle cesse définitivement en 1934. Des périodes plus ou moins longues d'abandon séparent les phases d'activité.

\section{LA PRODUCTION AU COURS DU HAUT-EMPIRE (I ${ }^{\text {er }}$ S.-DÉBUT DU III ${ }^{e}$ S.)}

Elle paraît être la plus importante en volume. La majeure partie de la carrière à ciel ouvert semble avoir été exploitée durant cette période. Les extractions les plus anciennes sont caractérisées par les traces d'escoude ${ }^{*}$ à 
double dent, formant des tranchées étroites à bords subverticaux ; l'arrachement du bloc se fait par emboîtures*.

La production est essentiellement tournée vers l'extraction de blocs de grand et moyen appareil. Quelques tambours de colonnes ou éléments de chapiteaux (4 exemplaires) peuvent être identifiés par leur forme cubique. Aucun enlèvement de fût de colonne n'est visible, mais seuls les fronts de taille ${ }^{*}$ et les ressauts de front de taille ont fait l'objet d'observation. Il n'est donc pas exclu qu'une fabrication de ce type ait pu être entreprise sur le sol de carrière principal.

Pour cette période, il n'est pas encore possible de définir si les éléments extraits recevaient une première ébauche en carrière ou s'ils étaient exportés " bruts de carrière " et finis sur le lieu de destination. Il est raisonnable de penser qu'ils étaient au moins grossièrement préparés pour alléger la charge à transporter. Cela a dû même aller au-delà du simple épannelage* des faces.

Une ébauche de personnage sculpté, mis au jour sur une laisse de carrière* (zone centrale carrière 1), témoigne de la présence de tailleurs de pierre et de sculpteurs sur le site, entre le $\mathrm{I}^{\mathrm{er}}$ et le $\mathrm{III}^{\mathrm{e}}$ s. (fig. 99).

\section{LA PRODUCTION DU BAS-EMPIRE (III ${ }^{\mathrm{e}}-\mathrm{V}^{\mathrm{e}} \mathrm{S}$.)}

Les observations n'ont porté que sur les parties visibles de la carrière 1 , essentiellement les fronts de taille $^{*}$ des exploitations en galerie (salles A à E).

L'extraction de sarcophages de plan rectangulaire est très largement dominante. C'est presque la totalité de la production de la salle B, dite salle souterraine (fig. 96). Les travaux semblent être planifiés car il y a uniformité de l'alignement des arrachements sur le front de taille* où un commencement d'extraction d'un bloc vertical de $230 \mathrm{~cm}$ de haut et de $40 \mathrm{~cm}$ de large semble correspondre à un fût de colonne ou à un pilastre. L'arrachement d'un bloc cubique de $65 \mathrm{~cm}$ d'arête marque l'extraction d'un élément destiné à être transformé en chapiteau ou en tambour de colonne.

Des éléments quadrangulaires $(40 \mathrm{~cm}$ environ dans leur plus grande dimension) ont été abandonnés dans les déblais de cette salle. Ils sont peut-être les indices d'un débitage de blocs de dimensions plus importantes, coffre de sarcophage par exemple, inadaptés à leur destination première.

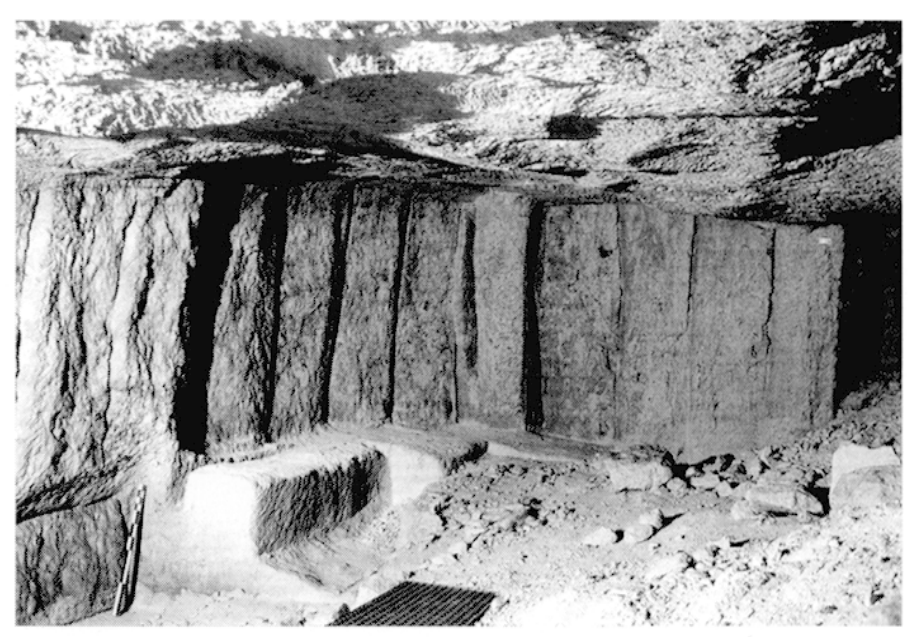

Fig. 96 - Angle sud-est du front de taille de la salle B dite salle souterraine. Empreintes des enlèements de convercles de sarcophage. en front de taille et de cuves de sarcophage sur sol de carriere. Le bloc vertical non extrail est destiné à un pilier ou une colonne. Au sol, l'extraction d'une cuve est restée inacheouée (photo F. Cognot, Maison de l'archéologie, Mâcon).

Les blocs sont découpés à l'escoude à tranchant droit moyen de 3,2 à $3,5 \mathrm{~cm}$ de large (fig. 96). Le front de taille (230 cm de hauteur) progresse par l'enlèvement de blocs destinés aux couvercles de sarcophages. Les cuves sont généralement découpées sur le sol de carrière libéré par l'avancée du front. L'arrachement se fait par forçage des coins de fer dans une encoignure ${ }^{*}$ horizontale à la base du bloc (cuve de sarcophage) ou verticale (couvercle de sarcophage).

Les cuves de sarcophages sont dégrossies sur place. Les faces sont rectifiées et l'intérieur évidé à l'escoude à tranchant droit moyen ou au ciseau. Des fragments de coffres laissés dans les rebuts montrent l'utilisation de la scie à dents. L'épaisseur des parois au sommet de la cuve est en moyenne de $5,7 \mathrm{~cm}$, donc bien moindre que dans les exemplaires en grès ( 10 à $12 \mathrm{~cm}$ ) de même époque. Le transport de cuves en calcaire à l'épaisscur aussi mince aurait été trop délicat si les cuves avaient été totalement évidées en carrière. Elles ne scront donc ébauchées et creusées que pour les alléger et faciliter leur transport. Elles seront finies sur le lieu de livraison, proche de la zone sépulcrale.

\section{LA PRODUCTION DU HAUT MOYEN ÂGE (VI'- -IX $^{\mathrm{e}} \mathrm{S}$.)}

C'est la suite logique, intensifiée, de la production de sarcophages. Ils sont de plan nettement trapézoïdal. 


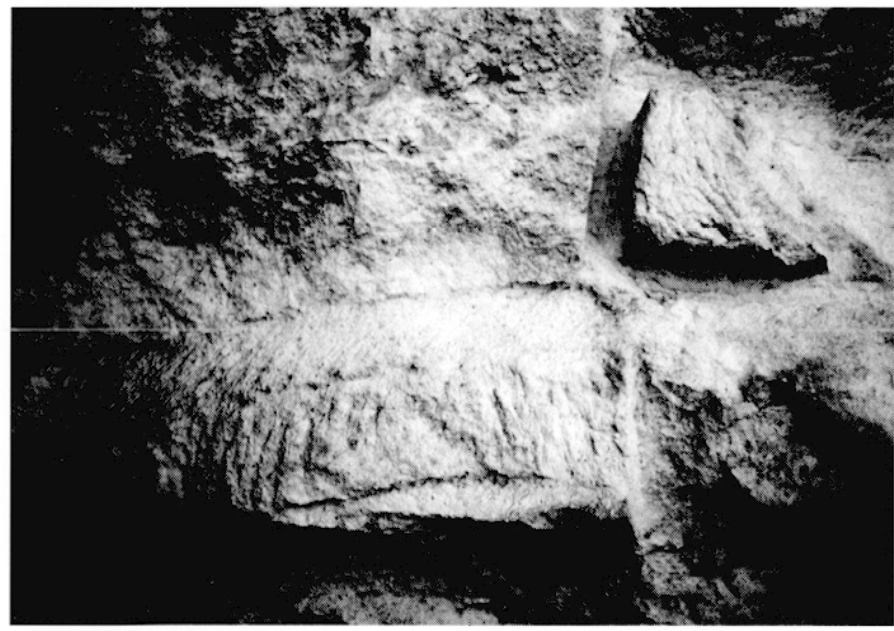

Fig. 97 - Empreintes d'extraction de couvercle en ciel de carrière de la salle A, alignées sur une diaclase (photo F. Cognot, Maison de l'archéologie, Mâcon).

Certaines extractions sont en " tête-bêche ", avec alternance d'une extraction à l'autre de la grande et la petite base en limite supérieure de front de taille*. Les empreintes de ces arrachements couvrent les fronts de taille et les sols de carrière apparents des salles A (dite grande cavité), C (dite petite cavité), D et E. Dans la majorité des cas, il y a reprise d'exploitation sur les fronts de taille du Bas-Empire. Des fragments de céramique mérovingienne ont été mis au jour en surface de la couche de comblement des fronts de taille (salle D et E) et dans une couche recouverte par des déchets de taille postérieurs (salle A).

La découpe des blocs est pratiquée au pic à pointe pyramidale, l'arrachement par coins insérés dans des encoignures* verticales pour les couvercles en front de taille, horizontales pour les coffres au sol. L'avancée des fronts, comme dans la phase précédente, détache essentiellement des couvercles. Les cuves sont généralement prélevées sur le sol dégagé. Quelques productions annexes peuvent apparaître çà et là (tentative d'extraction de colonnettes par exemple), mais ne sont que très sporadiques. Des blocs-couvercles (fig. 97) ont été également arrachés du ciel de carrière*. Cuves et couvercles ont une longueur moyenne de $220 \mathrm{~cm}$ et unc largeur de $80 \mathrm{~cm}$ (grande base) à $60 \mathrm{~cm}$ (petite base).

De nombreuses " alcôves " ou alvéoles de chantier rompent l'uniformité d'alignement du front de taille*. L'analyse des traces d'outil (sillons latéraux, lignes d'impact au fond des tranchées d'extraction*, etc.) dévoile une cohérence d'extraction dans chaque alcôve. Toutefois, la maîtrise du métier de carrier varie fortement d'une alcôve à une autre. Les travaux sont l'ouvre de petites équipes (2 à 3 personnes) qui sont intervenues indépendamment les unes des autres ou qui se sont succédé au cours de cette période d'extraction. Les défauts naturels (diaclases subverticales) sont mis à profit et constituent souvent des limites provisoires de front de taille. Il n'est pas encore possible d'établir si l'extraction précédait ou suivait la commande de sarcophages.

\section{LA PRODUCTION MÉDIÉVALE (X'X'XIVe S.)}

L'observation des techniques d'extraction et des traces des outils employés laisse supposer qu'une activité extractive assez intense s'est développée durant cette période d'essor des constructions religieuses et militaires. On sait que les abbés de Cluny se sont fournis en pierre de La Lie pour réaliser des colonnes et des chapiteaux décorés (Blanc et al., 1988). Dans la partie sud, et peut-être également sur le front de taille ${ }^{*}$ septentrional de la carrière 1, la production de blocs en grand appareil et de chapiteaux de colonnes (cube de $70 \mathrm{~cm}$ d'arête) pourrait être attribuable à cette période. La découpe se fait par grandes et larges tranchées sur sol de carrière ou sur les ressauts de front de taille. L'arrachement du bloc est réalisé par emboîtures*.

\section{LA PRODUCTION MODERNE (XVe-XVIII ${ }^{\mathrm{e}} \mathrm{S}$.)}

Si aucune trace dans la carrière ne permet d'appréhender le niveau et les types de production, des informations externes fournissent quelques indices sur l'activité de cette période. De nombreux éléments d'architecture civile ou religieuse, en pierre de La Lie, datables de cette époque, nous sont parvenus. Un document écrit mentionne que des groupes d'individus ou des familles habitaient ou avaient trouvé refuge dans les carrières. De là, ils perturbaient la vie des habitants des hameaux voisins. Ces groupes ou familles ont pu, à l'occasion, tirer profit de blocs mis au rebut par les exploitations antérieures et vendre cette production. 


\section{LA PRODUCTION CONTEMPORAINE (XIX ${ }^{\mathrm{e}} \mathrm{XX}^{\mathrm{e}} \mathrm{S}$.)}

Caractérisée par l'emploi systématique du "pétard" à la poudre noire, propre à obtenir du " caillou ", clle est éminemment destructive. Peu de fronts de taille ${ }^{*}$ encore apparents ont échappé aux foudres productives. Les fronts sud de la carrière en portent encore des traces et il est possible que cette " industrie " ait détruit en partie la voûte de la salle A, dite grande cavité, et en ait fait reculer le porche de plusieurs mètres. La technique d'extraction consiste à forer des trous, circulaires ou triangulaires convexes, de 50 à $80 \mathrm{~cm}$ de profondeur à l'aide d'un crayon de carrier*. Ils sont bourrés de poudre et allumés à la mèche. L'explosion fracture la roche en fragments informes plus ou moins volumineux et produit beaucoup de déchets.

Toute activité extractive, y compris celle de prélèvement de déblais, cesse entre 1934 et 1994.

\section{LA DIFFUSION DE LA PRODUCTION}

Une (ou plusieurs) bretelle (compendium) devait permettre l'exportation des productions des carrières de La Lie et rejoindre le réseau routier principal (voie Mâcon/ Autun, voie Mâcon/Moulins, voie Crêches-sur-Saône/ Autun par le " chemin des Allemands »). Aucune n'a été identifiée avec certitude. Il existe des chemins actuellement à usage agricole et forestier qui passent à proximité des fronts de carrière est et nord. Mais rien ne prouve qu'ils étaient déjà établis à l'époque romaine ou qu'ils étaient destinés à l'exportation du produit des carrières.

Les découvertes lithiques en " pierre de La Lie " sont autant d'indices permettant de discerner l'aire de répartition des productions de la carrière dans la région mâconnaise. Mais il faut, sur ce point, être très prudent. En effet, les carrières de La Lie n'ont pas eu le monopole de la production de ce type de calcaire. Ne serait-ce que dans la région proche, il a existé d'autres carrières dont le calcaire est très voisin, voire similaire : carrière de La Sénétrière (commune d'Hurigny), carrière 1 de Flacé-lès-Mâcon, carrière 1 de Sennecé-lès-Mâcon pour n'en mentionner que quelques-unes. Hors de son contexte, il devient parfois impossible de déterminer la provenance exacte du matériau.

Les blocs extraits des carrières de La Lie ont alimenté en priorité la demande locale. Le franchissement d'obstacles naturels (colline, rivière) accroît la difficulté du transport. La distance à parcourir allonge le délai de livraison et le coût du transport. Certaines productions ont pu être expédiées hors région, par la Saône, mais il faut considérer que ce ne sont là que des épiphénomènes.

L'écoulement principal de la production s'est fait " naturellement » par, et dans, la vallée inférieure de la Petite Grosne dont l'embouchure à Varenneslès-Mâcon est proche d'un gué antique. Des éléments provenant des carrières et datables de l'époque romaine ont été signalés sur les communes de Prissé, Chaintré, Vinzelles. La majeure partie des 300 éléments architecturaux romans ou postérieurs (XIII"-XVIII" s.) en " pierre de Ia Lie » est recensée dans cette vallée, au sud des carrières.

Une autre partie de la production, relativement importante, a été dispersée en direction du nord. Les éléments répertoriés jalonnent la "voie romaine " qui, venant de Prissé, rejoint la voie Mâcon/Autun vers SaintGengoux-de-Scissé. De nombreux exemplaires (essentiellement XIV'-XVIII"s.) se localisent sur les communes de Verzé, Igé, Azé. À Blanot, au moins deux sarcophages mérovingiens ont été brisés et ont été réemployés dans le prieuré, dépendance de Cluny.

Une troisième partie est dirigée vers l'ouest (Berzé-laVille, Berzé-le-Châtel, jusqu'à au moins Cluny). Il est actuellement difficile d'en estimer le volume.

Une dernière partie, malgré la difficulté de franchissement de la colline de Charnay-lès-Mâcon, a approvisionné Mâcon depuis l'époque romaine jusqu'à une date récente en concurrence avec la carrière de La Sénétrière à Hurigny (blocs en remploi du castrum du III ${ }^{*}$ s., sarcophages mérovingiens de l'église SaintClément, éléments d'architecture médiévale du vieux Saint-Vincent, éléments modernes de l'hospice de la Providence, etc.).

\section{LES CARRIÈRES : LIEU D'EXPRESSION, LIEU DE CROYANCE}

Lieux de travail et lieux de vie, les carrières sont aussi lieux de mémorisation de l'activité quotidienne, lieux d'expression de croyances. Les carrières de La Lie n'échappent pas à cette règle. Fronts de taille* et parfois sols en conservent encore les traces. 


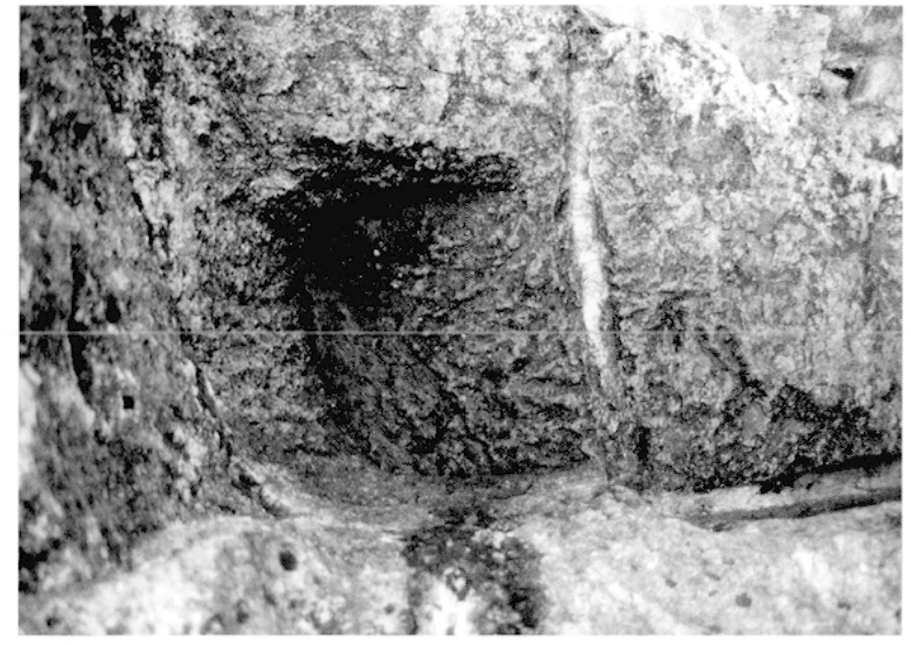

Fig. 98 - Traces d'appui d'un engin de levage, partie sommitale du front de carrière à ciel ouvert, zone sud de la carrière 1 (photo F. Cognot, Maison de l'archéologie, Mâcon).

\section{LES TRACES ACCOMPAGNATRICES DE L'ACTIVITÉ EXTRACTIVE}

\section{LES IMPLANTATIONS D'ENGINS DE LEVAGE}

Sur un front de taille de la carric̀re 1, dans le secteur sud, des implantations d'engins de levage semblent avoir été aménagées. En effet, un évidement circulaire et un autre pseudo-ellipsoïdal (fig. 98) ont été creusés dans et sur la partic supéricure d'un front de taille. Leur emplacement au-dessus de la zone d'extraction gallo-romaine, leur forme et leur dimension indiquent qu'un mât de charge a pu être installé. Il permettait de déplacer les blocs extraits à l'intérieur de la carrière mais aussi de les évacuer au-delà et au-dessus du front de carrière*, vers une aire de dégrossissage, un quai d'embarquement, une aire d'attente et de stockage de la production ou sur un véhicule de transport. La position de chacun des évidements laisse supposer, mais cela reste à vérifier, deux époques d'utilisation distinctes.

D'autres implantations d'engins ont été également inventées, toujours dans la carrière 1 , lors de nettoyages de fronts de taille. L'une, sur le front de taille nord, ne pcut être pour l'instant associée à une période d'exploitation précise, l'autre, sur le front ouest, a été découverte récemment (campagne 2000), dans un contexte gallo-romain tardif (traces d'escoude à tranchant plat moyen).

\section{PoINTS D'APPUI DE SOLIVES \\ ET TRACES D'HABITAT PRÉCAIRE, FORGE}

\section{Points d'appui de solives et traces d'habitat précaire}

Des creusements ménagés dans les fronts de taille à ciel ouvert sont indubitablement destinés à recevoir des solives ou des madriers. Ils sont très généralement rétrécis en fond par abaissement de la face supérieure. Ce sont des supports de toiture de structures légères ayant disparu.

Sur le front est, entre la salle A et la salle C, il en existe six séries étagées de deux à quatre unités. Il y a similitude dans l'ensemble des creusements formant une ligne d'appui. Des différences de section (carrée, rectangulaire, ronde, ovale) et de profondeur sont notables entre chaque ligne. Les lignes hautes sont généralement rondes ou ovales, les plus basses (à l'extérieur mais dans le prolongement du niveau du ciel de la salle $C$ ) sont systématiquement rectangulaires. Les « cabanes » successives n'auraient eu qu'un côté en appui, les autres côtés étant libérés de la paroi.

Un point d'appui (section formant les trois quarts d'un cercle de $13 \mathrm{~cm} \times 15 \mathrm{~cm}$, base horizontale, profondeur $8 \mathrm{~cm}$ ), est visible au sud de la salle $C$, jouxtant le bord droit d'un renfoncement du front est. Il paraît isolé, mais en examinant plus attentivement le bord gauche du renfoncement, un creusement semi-circulaire (base horizontale de $6 \mathrm{~cm}$, hauteur à l'ouverture $6 \mathrm{~cm}$, profondeur $5 \mathrm{~cm}$ ) réalisé au pic se laisse entrevoir. La " cabane " aurait ici deux côtés en appui, les deux autres étant libres, couvrant une aire de $300 \mathrm{~cm} \times 350 \mathrm{~cm}$.

Sur la paroi sud du front de taille ${ }^{*}$ sud de la carrière 1 , il existe un point d'appui creusé en limite supérieure du front de carrière* (section rectangulaire à face supérieure convexe, base horizontale de $14 \mathrm{~cm}$, hauteur $8 \mathrm{~cm}$, profondeur $14 \mathrm{~cm}$ ). Aucun autre point d'appui n'est visible sur le retour de front ouest partiellement détruit par des exploitations plus récentes. Il ne paraît d'ailleurs pas nécessaire ici, la solive pouvant être posée directement sur le sommet découvert du front existant à l'époque. En retrait du front, un empilement de blocs visiblement aménagé peut correspondre à une implantation du poteau d'angle de la " cabane " couvrant une aire approximative de $500 \mathrm{~cm} \times 400 \mathrm{~cm}$.

Dans la salle $\mathrm{C}$, des perforations de section carrée $(2 \mathrm{~cm} \times 2 \mathrm{~cm})$ ont été creusées à une dizaine de centi- 
mètres sous le ciel de la galerie, alignées dans le front de taille est (empreintes d'extraction de couvercles de sarcophages). Le mortier de chaux qui a enduit l'ensemble des parois ne les a pas recouvertes. Elles sont contemporaines du réaménagement de cette cavité à la fin du XVIII ${ }^{\mathrm{e}} \mathrm{s}$.

Contrairement aux carrières du Bois des Lens (Bessac, 1996), où les " cabanes "sont généralement proches ou en appui sur le front ouest, protégées des vents d'ouest et des pluies, dans les carrières de La Lie, aucune construction précaire n'est décelée sur le front ouest. Il faut cependant remarquer que ce front est presque totalement recouvert par les déchets de carrière ou par des apports extérieurs récents.

Forge

Dans la carrière 2 (partie sud du site), la commune, propriétaire, a fait prendre en 1999 des remblais pour " charger " des chemins de desserte. Au cours des travaux, des structures ont été partiellement détruites. La forte présence de charbons de bois et les éléments métalliques recueillis en surface présagent la présence d'une forge. Outre un bloc mis en rebut, de " tradition gallogrecque " (bandes parallèles sur les faces du bloc faites au taillant), les quelques tessons qui accompagnent charbons et fragments métalliques sont datables du début du I ${ }^{\text {er }} \mathrm{s}$. Les restes de ce qui pourrait être un atelier sont exposés depuis aux intempéries, attendant une hypothétique autorisation d'intervention.

\section{SCULPTURE}

Des stèles en pierre de La Lie ont été mises au jour dans le Mâconnais. L'une d'elles, découverte au siècle dernier à Solutré, est actuellement exposée au musée de Saint-Germain-en-Laye. Une autre, trouvée à Mâcon lors de fouilles récentes (Cognot, 1991), a enrichi les collections du musée des Ursulines. Au cours des nettoyages des fronts apparents, différents éléments sculptés sont apparus dans les carrières de La Lie.

\section{PersonNage sculPté}

Sur une laisse de carrière ${ }^{*}$, dans la zone centrale de l'exploitation à ciel ouvert, un nettoyage a révélé une

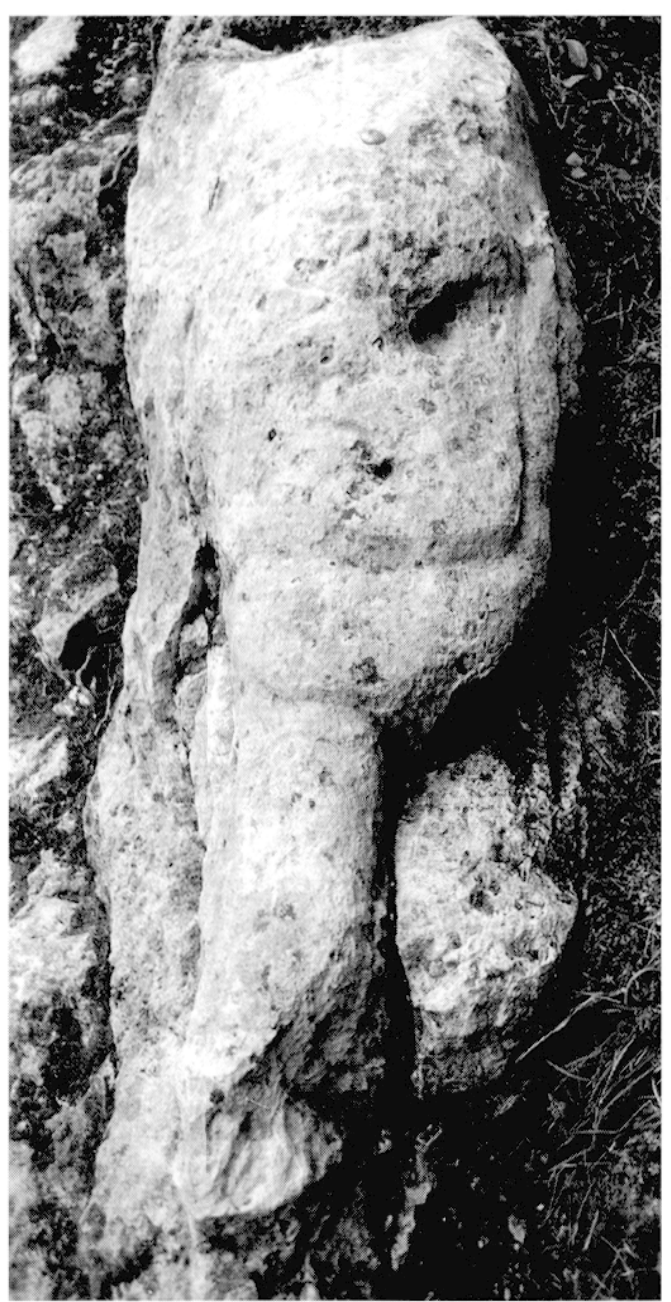

Fig. 99 - P'ersonnage sculplé sur une laisse de carrière (photo F. Cognot, Maison de l'archéologie, Mâcon).

sculpture en haut-relief (fig. 99). C'est plus une ébauche qu'un travail fini. Un personnage, nu ou à demi-vêtu, est allongé sur le dos, la fesse gauche légèrement débordante. À l'origine il mesurait $264 \mathrm{~cm}$ mais il n'en reste que $205 \mathrm{~cm}$. Les membres supérieurs ont disparu, ne restent que les membres inférieurs. Le droit cassé sous la pliure du genou, le gauche à mi-cuisse. La tête manque mais le contour se devine encore. Elle peut avoir été coiffée d'un bonnet phrygien mais les restes du contour de la coiffure se confondent avec ceux de la roche. Le personnage, gras et adipeux, laisse paraître un abdomen replet, asexué.

Sur le haut du buste et la place anciennement occupée par le cou, est gravé un blason dont se discerne encore l'écartelé. L'usure du temps en a fait disparaitre le remplissage et les motifs. Sur le côté gauche, au niveau de la taille, une amorce circulaire en relief, rattachée au 


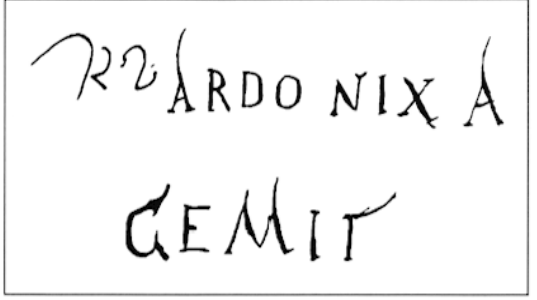

Fig. 100 - Inscription peinte : ardo nix a gemit (d'après A. Arcelin, 1881).

corps ou au vêtement, a été ciscléc. Sur l'abdomen, côté droit, quatre à cinq lettres de 6 à $7 \mathrm{~cm}$ de hauteur sont sommairement gravées. Elles sont fortement usées par les intempéries. Leur signification reste obscure.

Un moment considéré comme une représentation d'Attis dont il a la position allongée, l'absence de sexe, le bonnet phrygien, ce personnage sculpté n'en a ni la jeunesse ni la gracilité. Il serait plutôt à rapprocher des représentations de Silvain, qui jouissait de la ferveur des carriers, au même titre qu'Hercule Saxanus. Les traces d'outil qui entourent le personnage sont datables de la fin du $\mathrm{I}^{\mathrm{er}} \mathrm{s}$. (escoude ${ }^{*}$ à double dent).

Cette ébauche atteste la présence d'un sculpteur sur le site. Son atelier était-il installé à proximité ? La question reste en suspens. Seules des fouilles programmées sur la zone pourraient éventuellement apporter une réponse définitive.

\section{Cegur et Crodx}

Sur le front de taille* nord de la salle $\mathrm{C}$, dite petite cavité, un relief forme un cœur incluant une croix grecque. Au-dessus de ce premier motif, une autre croix grecque est également ciselée. Les parois de la salle $\mathrm{C}$ ont été enduites de mortier à la chaux; certains appuis de solives ont été agrandis; l'ouverture de la voûte a été fermée par un mur également enduit. Ces aménagements et les gravures associées montrent que l'espace a été transformé en lieu de séjour et peut-être lieu de culte. L'ensemble concerne la période révolutionnaire (1792-1796) el la fin de la période napoléonienne (1808-1811). Les parties souterraines de la carrière, encore accessibles à la fin du XVIII's., ont servi de refuge à des prêtres réfractaires puis à des jeunes hommes refusant d'être enrôlés dans l'arméc napoléonienne. Un arrêté du maire en 1810 ordonne aux habitants de boucher dans l'année les cavités refuges.

\section{AUTRES MARQUES GLYPTOGRAPHIQUES}

Sur un redan de front de taille, dans la zone sud de la carrière 1 , une croix latine, à la branche inférieure pattée, est gravée en creux. Ce peut être une croix d'exorciste comme il en existe de nombreux exemplaires dans la région. Difficilement datables, ces symboles sont encore communément reproduits au XIX"s.

Sur le même redan, à un niveau inférieur, un motif incisé à champlever, arrondi et convexe, ne peut être interprété. Il semble avoir été légèrement mutilé par l'extraction postéricure d'un bloc.

Une croix grecque est gravée sur un bloc erratique, dans le sud de la carrière (zone sud, partie ouest).

\section{PERFORATION}

Sur le front de taille* nord, un percement de part en part joint les deux côtés d'un angle saillant. Cette perforation a été réalisée au ciseau ou au foret. On peut supposer qu'elle a pu servir de passant pour une corde, soit pour une extraction, soit, et c'est l'hypothèse actuellement retenue, pour attacher un animal de trait (bouf).

\section{INSCRIPTION PEINTE}

Quelques rares traces de peinture rouge (ou de minium) sont visibles sur le front de taille*, à l'extrême sud de la carrière 1 . En attendant de disposer des moyens techniques permettant leur conservation hors sol, elles ont de nouveau été enfouies sous une couche protectrice de remblais. Une étude récente des espèces végétales croissant dans les carrières a montré la présence de plusieurs pieds de garance. Cette espèce a été manifestement importée. Le lien entre la plante et le colorant rouge utilisé pour les inscriptions n'est pas encore établi.

Une autre inscription peinte sur un front de taille des carrières de La Lie a été relevée par Adrien Arcelin en 1854 (fig. 100). Elle a fait l'objet d'une traduction (Arcelin, 1881, p. 300). D'après cet auteur, Ardonixa serait " un nom féminin d'origine gauloise".

$$
\text { * * }
$$

Les études entreprises depuis 1994 sur les carrières de Ia Lie n'ont porté que sur les ćléments visibles et acces- 
sibles du site. Les autorisations délivrées, y compris celle courant jusqu'en 2002, interdisent radicalement toute atteinte au sol et sous-sol.

L'épaisse couche de remblais rend actuellement impossible l'étude des sols de carrière tant dans la partie exploitée à ciel ouvert que dans les parties exploitées en galeries. Cette étude est pourtant indispensable à la compréhension générale du site et à la chronologie des exploitations.

Les résultats, limités par toutes ces contingences, ont cependant permis de distinguer de grandes phases d'exploitation. Du simple bloc destiné à l'élévation de murs aux cuves et couvercles de sarcophages, du tambour de colonne au chapiteau, de l'auge commune aux sculptures votives, l'éventail des productions reconnues dans les carrières de La Lie, déjà large, n'est peut-être pas complet.

Les observations portant sur les évolutions et les transformations chronologiques des outils d'extraction et de taille, ainsi que les mutations des méthodes d'extraction corroborent les observations faites dans les carrières méditerranéennes (Bessac, 1996). Les outils et les techniques mis en ceuvre lors du façonnage après extraction peuvent être comparés à ceux mis en évidence sur les sites méditerranéens. La taille brochée (ou piquée) et le sciage (scie à dents) sont pratiqués. L'emploi du marteau taillant " gallo-grec ", en larges lignes parallèles (tranchant de $80 \mathrm{~mm}$ ) sur les faces d'un bloc, permet de situer les débuts de l'exploitation des carrières de La Lie aux environs de la dernière décennie du $\mathrm{I}^{\mathrm{er}} \mathrm{s}$. avant J.-C.

Deux différences notoires apparaissent dans la comparaison des productions: dans l'état actuel des connaissances, il ne semble pas qu'on ait extrait à La Lie de blocs destinés à de grandes colonnes monolithiques au cours de la période gallo-romaine, ni de cylindres par tranchée circulaire (empreinte ronde) au cours du Moyen Âge. Toutefois, ces extractions sont pratiquées en majorité sur sol de carrière (Bessac, 1996), or celui-ci est actuellement totalement inaccessible à La Lie.

Des indices nombreux et concordants emportent la conviction qu'il y a eu occupation humaine et structuration de l'espace: atelier de taille et de sculpture, habitat précaire, forge, étable.

La production (et le marché) de la pierre de taille, longtemps négligée, se révèle être un indicateur du niveau de développement technique, économique, social et culturel. Avec des fluctuations moins prononcées et une évolution plus lente, elle est, quelque part, comparable à la production (et au marché) de la céramique. Pour le caractériser, cet indicateur est élaboré à partir d'observations portant sur les méthodes et l'organisation de l'extraction, l'évolution de la forme de l'outil, la variation de la demande en pierre de taille, la variation de la destination de la production cultuelle, civile ou militaire.

L'étude des carrières de La Lie participe aux recherches sur la production (et le marché) de la pierre de taille. Les premiers résultats confortent les conclusions portant sur d'autres carrières - carrière de Saint-Boil, Saône-et-Loire (Monthel, Pinette, 1977 et Monthel, supra, p. 89-120), carrières du Bois des Lens, Gard (Bessac, 1996 et Bessac, supra, p. 29-51) - ou sur des ouvrages plus généraux (Adam, 1984; Bedon, 1984; Bessac et al., 1999). Bien des années de recherches, accompagnées des moyens adaptés, sont encore nécessaires avant de disposer d'informations suffisamment pertinentes à la connaissance réelle du site et de son apport notable dans le domaine de l'archéologie de la pierre. 